Bull. Korean Math. Soc. 48 (2011), No. 1, pp. 35-50

DOI 10.4134/BKMS.2011.48.1.035

\title{
STABLE INDEX PAIRS FOR DISPERSIVE DYNAMICAL SYSTEMS
}

\author{
Yoon Hoe Goo And Jong-Suh Park
}

\begin{abstract}
We construct the index pairs of an isolated neighborhood for a dispersive dynamical system and investigate the existence of an index pair which is stable under small perturbations of the dispersive dynamical systems.
\end{abstract}

\section{Introduction}

The Conley index theory, the origin of which goes back to the famous Wazewski Retract Theorem [9], has become an important tool in the qualitative study of differential equations. The theory provides cohomological or homotopic invariants of isolated invariant sets of flows and yields existence results in differential equation. The foundations of the theory, in a locally compact setting, were established by R. Churchil [1], J. Montgomery [11], C. Conley [2], and by H. Kurland [9]. K. Rybakowski [13] extended the theory to the case of non-locally compact spaces. The theory is now designated as the Conley index theory because of the significant role played by $\mathrm{C}$. Conley in its development.

There is a formal similarity between the above indices of the isolated invariant set and the fixed point index [4] of a continuous map. Recently the fixed point index has been extended to the case of a multi-valued admissible map [8]. Thus there is a question if a similar generalization is also possible in case of a general dynamical system.

Such a generalization would be useful in direct applications to differential equation without uniqueness as well as in situations where the classical Conley index is used in course of a proof but extra assumptions or extra verifications are needed to ensure uniqueness [10].

Received May 7, 2009

2010 Mathematics Subject Classification. Primary 37B30; Secondary 54H20.

Key words and phrases. index pair, dispersive dynamical systems, $I$-solution, isolating neighborhood.

This work was supported by the research fund from Hanseo University in 2008.

(C)2011 The Korean Mathematical Society 
E. Dancer mentioned in [3] that an approximation technique could provide a partial extension of the Conley theory to the case of general dynamical systems defined by differential equations without uniqueness.

In this paper we construct the index pairs of an isolated invariant set of a dispersive dynamical system on a metric space. Such a topological approach, contrary to the approximation technique, seems to be closer in spirit to the original Conley index. Additionally it allows applications to differential inclusions.

Our work is organized as follows. In Section 2, we study some properties of I-solution for dispersive dynamical systems. In the following section we investigate the existence and properties of index pairs. In the last section we provide that the index pairs are stable under small perturbations of the dispersive dynamical system.

\section{General dynamical systems}

Let $X$ be a topological space. We will denote by $2^{X}$ the set of all nonempty compact subsets of $X$.

Definition 2.1. Let $(X, d)$ and $(Y, \rho)$ be metric spaces and let $f: X \rightarrow 2^{Y}$. The map $f$ is said to be (1) upper semicontinuous (usc) at $x \in X$ if for each $\varepsilon>0$, there exists $\delta>0$ such that

$$
d(x, z)<\delta \text { implies } f(z) \subseteq B_{\rho}(f(x), \varepsilon),
$$

(2) lower semicontinuous (lsc) at $x$ if for each $\varepsilon>0$, there exists $\delta>0$ such that

$$
d(x, z)<\delta \text { implies } f(x) \subseteq B_{\rho}(f(z), \varepsilon) .
$$

The map $f$ is said to be continuous at $x$ if $f$ is upper and lower semicontinuous at $x$.

It is easy to prove the following proposition.

Proposition 2.2. Let $(X, d)$ and $(Y, \rho)$ be metric spaces and let $f: X \rightarrow 2^{Y}$. Then

(1) $f$ is usc at $x \in X$ if and only if for any open neighborhood $U$ of $f(x)$, there exists an open neighborhood $V$ of $x$ such that $f(z) \subset U$ for all $z \in X$.

(2) $f$ is lsc at $x \in X$ if and only if for any open set $U$ with $f(x) \cap U \neq \emptyset$, there exists an open neighborhood $V$ of $x$ such that $f(z) \cap U \neq \emptyset$ for all $z \in V$.

Proposition 2.3. Let $(X, d)$ and $(Y, \rho)$ be metric spaces and let $f: X \rightarrow 2^{Y}$.

(1) If $f$ is usc at $x \in X$, then $x_{n} \rightarrow x, y_{n} \in f\left(x_{n}\right)$ and $y_{n} \rightarrow y$ implies $y \in f(x)$.

(2) If $X$ is compact, then the inverse of (1) holds.

Proof. (1) Suppose $f$ is usc at $x \in X$ and let $\varepsilon>0$. Then there exists $\delta>0$ such that $d(x, z)<\delta$ implies $f(z) \subset B_{\rho}\left(f(x), \frac{\varepsilon}{2}\right)$. Also, there exists a positive integer $n$ such that $d\left(x, x_{n}\right)<\delta$ and $d\left(y, y_{n}\right)<\frac{\varepsilon}{2}$. Therefore we have $y_{n} \in$ 
$f\left(x_{n}\right) \subset B_{\rho}\left(f(x), \frac{\varepsilon}{2}\right)$ and so there exists $z \in f(x)$ such that $\rho\left(y_{n}, z\right)<\frac{\varepsilon}{2}$. Since $\rho(y, z) \leq \rho\left(y, y_{n}\right)+\rho\left(y_{n}, z\right)<\frac{\varepsilon}{2}+\frac{\varepsilon}{2}=\varepsilon$, we have $z \in B_{\rho}(y, \varepsilon) \cap f(x)$. Hence it follows that $y \in \overline{f(x)}=f(x)$.

(2) Suppose $f$ is not usc at $x \in X$. Then there exists $\varepsilon>0$ such that for any $\delta>0$, there exists $z \in B_{d}(x, \delta)$ such that $f(z) \nsubseteq B_{\rho}(f(x), \varepsilon)$. Therefore for any positive integer $n$, there exists $x_{n} \in B_{d}\left(x, \frac{1}{n}\right)$ such that $f\left(x_{n}\right) \nsubseteq B_{\rho}(f(x), \varepsilon)$. Also there exists $y_{n} \in f\left(x_{n}\right)-B_{\rho}(f(x), \varepsilon)$. Since $X$ is compact, $\left(y_{n}\right)$ has a convergent subsequence. Let $y_{n} \rightarrow y$. Since $x_{n} \rightarrow x$, we have $y \in f(x)$. But since $\rho\left(y_{n}, f(x)\right) \geq \varepsilon$ for all $n$, we have $\rho(y, f(x)) \geq \varepsilon$. This is a contradiction. Hence $f$ is usc at $x \in X$. This completes the proof.

Continuing on in the same vein, we state another condition that is equivalent to upper semicontinuity.

Proposition 2.4. Let $(X, d)$ and $(Y, \rho)$ be metric spaces and let $f: X \rightarrow 2^{Y}$. Then $f$ is usc if and only if for any open set $U \subset Y, f^{-1}(U) \equiv\{x \in X \mid f(x) \subset$ $U\}$ is open in $X$.

Proof. Suppose $f$ is usc. Then for any $x \in f^{-1}(U)$, we have $f(x) \subset U$. By Proposition 2.2, there exists a neighborhood $V$ of $x$ such that $z \in V$ implies $f(z) \subset U$. Therefore we have $V \subset f^{-1}(U)$ and so $f^{-1}(U)$ is open in $X$.

Conversely, let $x \in X$ and let $U$ be any open neighborhood of $f(x)$. Then $V \equiv f^{-1}(U)$ is a neighborhood of $x$ and for any $z \in V$ we have $f(z) \subset U$. By Proposition 2.2, $f$ is usc at $x$. Hence $f$ is usc and so the proof is complete.

Proposition 2.5. Let $f: X \rightarrow 2^{Y}$ be usc. If $K$ is a compact subset of $X$, then $f(K)$ is a compact subset of $Y$.

Proof. Let $\left\{U_{\alpha} \mid \alpha \in A\right\}$ be an open cover of $f(K)$. For each $x \in K$, since $f(x)$ is compact, there exists a finite subset $A_{x}$ of $A$ such that $f(x) \subset \bigcup_{\alpha \in A_{x}} U_{\alpha}$. Since $f$ is usc, there exists a neighborhood $V_{x}$ of $x$ such that $f\left(V_{x}\right) \subset \bigcup_{\alpha \in A_{x}} U_{\alpha}$. Since $K$ is compact, there are finitely many $x_{1}, \ldots, x_{n} \in K$ such that $K \subset \bigcup_{i=1}^{n} V_{x_{i}}$. Then

$$
f(K) \subset f\left(\bigcup_{i=1}^{n} V_{x_{i}}\right)=\bigcup_{i=1}^{n} f\left(V_{x_{i}}\right) \subset \bigcup_{i=1}^{n} \bigcup_{\alpha \in A_{x_{i}}} U_{\alpha} .
$$

Thus $f(K)$ is compact.

We denote the set of all real numbers, nonnegative real numbers, and nonpositive real numbers by $\mathbb{R}, \mathbb{R}^{+}$and $\mathbb{R}^{-}$, respectively.

Definition 2.6. An usc mapping $f: X \times \mathbb{R} \rightarrow 2^{X}$ is called a dispersive dynamical system if the following conditions are satisfied:

(1) For all $x \in X, f(x, 0)=\{x\}$;

(2) For all $s, t \in \mathbb{R}$ with $s t \geq 0$ and all $x \in X, f(f(x, s), t)=f(x, s+t)$;

(3) For all $x, y \in X$, if $y \in f(x, t)$, then $x \in f(y,-t)$. 
The sets $f(\{x\} \times \mathbb{R}), f\left(\{x\} \times \mathbb{R}^{+}\right), f\left(\{x\} \times \mathbb{R}^{-}\right)$will be called the trajectory, the positive trajectory and the negative trajectory of $x$ and denoted by $f(x), f^{+}(x), f^{-}(x)$, respectively.

In the following argument, all of the notations and results are stated with respect to a given dispersive dynamical system $f$ defined on a metric space $X$.

Definition 2.7. Let $I \subset \mathbb{R}$ be an interval. By a $I$-solution of $f$ in $N \subset X$ we mean a continuous map $\sigma: I \rightarrow N$ such that

$$
\sigma(t) \in f(\sigma(s), t-s) \text { for all } s, t \in I \text {. }
$$

We will say that the solution $\sigma$ originates at $x \in X$ if $0 \in I$ and $\sigma(0)=x$. A $[0, t]$-solution $([t, 0]$-solution in case $t \leq 0) \sigma$ of $f$ in $N$ will be called a $t$ connection from $x$ to $y$ provided $\sigma(0)=x, \sigma(t)=y$. The set of all $I$-solution of $f$ in $N$ and the set of all $I$-solutions originating at $x$ will be denoted by $\operatorname{sol}_{N}(I), \operatorname{sol}_{N}(I, x)$, respectively. We will also write $\operatorname{conn}_{N}(t, x, y)$ for the set of all $t$-connections from $x$ to $y$ in $N$. In case $N=X$ the subscripts $N$ in $s_{N}$ and $\operatorname{conn}_{N}$ will be omitted.

We have the following basic facts $[3,4,6,7,8,9,10]$.

Proposition 2.8. If $\sigma_{1}$ and $\sigma_{2}$ are solutions of $f$ on $[a, b]$ and $[b, c]$, respectively, with $\sigma_{1}(b)=\sigma_{2}(b)$, then the concatenation $\sigma$ of $\sigma_{1}$ and $\sigma_{2}$ defined by

$$
\sigma(t)= \begin{cases}\sigma_{1}(t), & t \in[a, b] \\ \sigma_{2}(t), & t \in[b, c]\end{cases}
$$

is a solution of $f$ on $[a, c]$.

Proposition 2.9. Let $y \in f\left(x, t_{1}-t_{0}\right)$, where $t_{0} \leq t_{1}$. Then there exists $a$ solution $\sigma:\left[t_{0}, t_{1}\right] \rightarrow X$ such that $\sigma\left(t_{0}\right)=x, \sigma\left(t_{1}\right)=y$.

Proposition 2.10 (Barbashin's Theorem). Let $\left(\sigma_{n}\right)$ be a sequence of solutions $\sigma_{n}:\left[t_{0}, t_{1}\right] \rightarrow X$ and let $\sigma_{n}\left(t_{0}\right) \rightarrow x$. Then there exist a subsequence $\left(\sigma_{n_{i}}\right)$ of $\left(\sigma_{n}\right)$ and a solution $\tau:\left[t_{0}, t_{1}\right] \rightarrow X$ such that $\left(\sigma_{n_{i}}\right)$ converges uniformly to $\tau$ on $\left[t_{0}, t_{1}\right]$.

We shall investigative some properties of $I$-solution for a dispersive dynamical system $f$ which will be used in the next section.

Proposition 2.11. If $I, J$ are compact intervals such that $I \subset J$, then for every $I$-solution $\sigma$ there exists a J-solution $\tau$ being an extension of $\sigma$.

Proof. Let $I=[b, c]$ and $J=[a, d]$. Put $x_{0}=\sigma(b)$ and $x_{1}=\sigma(c)$. Choose $y_{0} \in f\left(x_{0}, b-a\right)$ and $y_{1} \in f\left(x_{1}, d-c\right)$. Since $x_{0} \in f\left(y_{0}, b-a\right)$, by Proposition 2.9 , there exist solutions $\gamma_{1}:[0, b-a] \rightarrow X$ and $\gamma_{2}:[0, d-c] \rightarrow X$ such that $\gamma_{1}(0)=y_{0}, \gamma_{1}(b-a)=x_{0}, \gamma_{2}(0)=x_{1}, \gamma_{2}(d-c)=y_{1}$. Define $\tau:[a, d] \rightarrow X$ by

$$
\tau(t)= \begin{cases}\gamma_{1}(t-a), & t \leq b \\ \sigma(t), & b \leq t \leq c \\ \gamma_{2}(t-c), & t \geq c .\end{cases}
$$

Then $\gamma$ is a solution being an extension of $\sigma$. 
Proposition 2.12. Assume $N \subset X$ is compact, and sequences $\left(x_{n}\right),\left(y_{n}\right) \subset$ $N,\left(t_{n}\right) \subset \mathbb{R}$ are convergent: $x_{n} \rightarrow x, y_{n} \rightarrow y, t_{n} \rightarrow t$. If $\operatorname{conn}_{N}\left(t_{n}, x_{n}, y_{n}\right) \neq \emptyset$, then $\operatorname{conn}_{N}(t, x, y) \neq \emptyset$.

Proof. First consider the case $t \geq 0$. There exists a sequence $\left(\sigma_{n}\right)$ of solutions $\sigma_{n}:\left[0, t_{n}\right] \rightarrow N$ such that $\sigma_{n}(0)=x_{n}, \sigma_{n}\left(t_{n}\right)=y_{n}$ for all $n$. Choose a number $T>t$. We may assume that $t_{n}<T$ for all $n$. By Proposition 2.11, there exists a sequence $\left(\tau_{n}\right)$ of solutions $\tau_{n}:[0, T] \rightarrow X$ being an extension of $\sigma_{n}$ for all $n$. By Proposition 2.10, there exist a subsequence $\left(\tau_{n_{i}}\right)$ of $\left(\tau_{n}\right)$ and a solution $\gamma:[0, T] \rightarrow X$ such that $\left(\tau_{n_{i}}\right)$ converges uniformly to $\gamma$ on $[0, T]$.

Let $0 \leq s<t$. We may assume that $s<t_{n_{i}}$ for all $i$. Since

$$
\gamma(s)=\lim _{i \rightarrow \infty} \tau_{n_{i}}(s)=\lim _{i \rightarrow \infty} \sigma_{n_{i}}(s),
$$

we have $\gamma(s) \in N$. Since $\gamma$ is continuous, $\gamma(t)=\lim _{s \rightarrow t^{-}} \gamma(s) \in N$. Thus we have

$$
\left.\gamma\right|_{[0, t]}:[0, t] \rightarrow N .
$$

We have $\gamma(0)=\lim _{i \rightarrow \infty} \tau_{n_{i}}(0)=\lim _{i \rightarrow \infty} \sigma_{n_{i}}(0)=\lim _{i \rightarrow \infty} x_{n_{i}}=x$. Given any $\varepsilon>0$, there exists $\delta>0$ such that if $|t-s|<\delta$, then $d(\gamma(t), \gamma(s))<\frac{\varepsilon}{3}$. Since $\left(\tau_{n_{i}}\right)$ converges uniformly to $\gamma$ on $[0, T], y_{n_{i}} \rightarrow y$ and $t_{n_{i}} \rightarrow t$, there exists $k$ such that

$$
d\left(\tau_{n_{k}}(s), \gamma(s)\right)<\frac{\varepsilon}{3} \text { for all } s \in[0, T], d\left(y_{n_{k}}, y\right)<\frac{\varepsilon}{3}, \text { and }\left|t-t_{n_{k}}\right|<\delta .
$$

Then we have

$$
\begin{aligned}
d(\gamma(t), y) & \leq d\left(\gamma(t), \gamma\left(t_{n_{k}}\right)\right)+d\left(\gamma\left(t_{n_{k}}\right), \tau_{n_{k}}\left(t_{n_{k}}\right)\right)+d\left(\tau_{n_{k}}\left(t_{n_{k}}\right), y\right) \\
& =d\left(\gamma(t), \gamma\left(t_{n_{k}}\right)\right)+d\left(\gamma\left(t_{n_{k}}\right), \tau_{n_{k}}\left(t_{n_{k}}\right)\right)+d\left(\sigma_{n_{k}}\left(t_{n_{k}}\right), y\right) \\
& =d\left(\gamma(t), \gamma\left(t_{n_{k}}\right)\right)+d\left(\gamma\left(t_{n_{k}}\right), \tau_{n_{k}}\left(t_{n_{k}}\right)\right)+d\left(y_{n_{k}}, y\right) \\
& <\frac{\varepsilon}{3}+\frac{\varepsilon}{3}+\frac{\varepsilon}{3}=\varepsilon .
\end{aligned}
$$

Thus $\gamma(t)=y$ and so $\left.\gamma\right|_{[0, t]} \in \operatorname{conn}_{N}(t, x, y)$.

The proof of the case $t<0$ is similar.

We conclude the section with the following proposition.

Proposition 2.13. Assume $N$ is compact, and $\left(x_{n}\right) \subset N$ is a sequence such that $x_{n} \rightarrow x$. Then

(a) Let $\left(t_{n}\right) \subset \mathbb{R}^{+}$be a sequence such that $\operatorname{sol}_{N}\left(\left[0, t_{n}\right], x_{n}\right) \neq \emptyset$ for all $n$. If $t_{n} \rightarrow \infty$, then $\operatorname{sol}_{N}\left(\mathbb{R}^{+}, x\right) \neq \emptyset$.

(b) Let $\left(t_{n}\right) \subset \mathbb{R}^{-}$be a sequence such that $\operatorname{sol}_{N}\left(\left[t_{n}, 0\right], x_{n}\right) \neq \emptyset$ for all $n$. If $t_{n} \rightarrow-\infty$, then $\operatorname{sol}_{N}\left(\mathbb{R}^{-}, x\right) \neq \emptyset$.

Proof. (a) We may assume that $t_{n}<t_{n+1}$ for all $n$. Let $\sigma_{n} \in \operatorname{sol}_{N}\left(\left[0, t_{n}\right], x_{n}\right)$. Since $\sigma_{n}(0)=x_{n} \rightarrow x$, by Proposition 2.10, there exist a subsequence $\left(\sigma_{n_{i}}\right)$ of 
$\left(\sigma_{n}\right)$ and a solution $\tau_{1}:\left[0, t_{1}\right] \rightarrow X$ such that $\left(\sigma_{n_{i}}\right)$ converges uniformly to $\tau_{1}$ on $\left[0, t_{1}\right]$. We have

$$
\tau_{1}(0)=\lim _{i \rightarrow \infty} \sigma_{n_{i}}(0)=\lim _{i \rightarrow \infty} x_{n_{i}}=\lim _{n \rightarrow \infty} x_{n}=x .
$$

Since $\tau_{1}(s)=\lim _{i \rightarrow \infty} \sigma_{n_{i}}(s) \in N$ for all $s \in\left[0, t_{1}\right]$, we have $\tau_{1}:\left[0, t_{1}\right] \rightarrow N$. We may assume that $n_{1} \geq 2$. Since $\sigma_{n_{i}}=x_{n_{i}} \rightarrow x$, by Proposition 2.10, there exist a subsequence $\left(\sigma_{n_{i_{k}}}\right)$ of $\left(\sigma_{n_{i}}\right)$ and a solution $\tau_{2}:\left[0, t_{2}\right] \rightarrow X$ such that $\left(\sigma_{n_{i_{k}}}\right)$ converges uniformly to $\tau_{2}$ on $\left[0, t_{2}\right]$. Clearly $\tau_{2}:\left[0, t_{2}\right] \rightarrow N$. Since $\tau_{2}(s)=\lim _{k \rightarrow \infty} \sigma_{n_{i_{k}}}(s)=\lim _{i \rightarrow \infty} \sigma_{n_{i}}(s)=\tau_{1}(s)$ for all $s \in\left[0, t_{1}\right]$, we have $\tau_{2}=\tau_{1}$ on $\left[0, t_{1}\right]$. Repeating this process, we obtain a sequence $\left(\tau_{n}\right)$ of solutions $\tau_{n}:\left[0, t_{n}\right] \rightarrow N$ such that $\tau_{n+1}=\tau_{n}$ on $\left[0, t_{n}\right]$ for all $n$. Define $\gamma: \mathbb{R}^{+} \rightarrow N$ by $\left.\gamma\right|_{\left[0, t_{n}\right]}=\tau_{n}$. Then $\gamma \in \operatorname{sol}_{N}\left(\mathbb{R}^{+}, x\right)$.

(b) It is similar to the proof of (a).

\section{Existence of index pairs and their properties}

In this section we investigate the existence of an index pair for an isolating neighborhood and its properties. Let $N$ be a compact subset of $X$. Define a $\operatorname{map} f_{N}: N \times \mathbb{R} \rightarrow 2^{N}$ by

$$
f_{N}(x, t)=\left\{y \in N \mid \operatorname{conn}_{N}(t, x, y) \neq \emptyset\right\} .
$$

Proposition 3.1. The mapping $f_{N}$ is a dispersive dynamical system.

Proof. Let $(x, t) \in N \times \mathbb{R}$. We may assume that $t \geq 0$. Let $\left(y_{n}\right)$ be a sequence in $f_{N}(x, t)$. For each positive integer $n$, there exists a solution $\sigma_{n}:[0, t] \rightarrow$ $N$ such that $\sigma_{n}(0)=x$ and $\sigma_{n}(t)=y_{n}$. Since $N$ is compact, $\left(y_{n}\right)$ has a convergent subsequence. Let $y_{n} \rightarrow y \in N$. By Barbashin's Theorem, there exist a subsequence $\left(\sigma_{n_{i}}\right)$ of $\left(\sigma_{n}\right)$ and a solution $\sigma:[0, t] \rightarrow X$ such that $\left(\sigma_{n_{i}}\right)$ converges uniformly to $\sigma$ on $[0, t]$. Since $\sigma(s)=\lim _{i \rightarrow \infty} \sigma_{n_{i}}(s) \in N$, we have $\sigma:[0, t] \rightarrow N$. Since

$$
\sigma(0)=\lim _{i \rightarrow \infty} \sigma_{n_{i}}(0)=x \text { and } \sigma(t)=\lim _{i \rightarrow \infty} \sigma_{n_{i}}(t)=\lim _{i \rightarrow \infty} y_{n_{i}}=y,
$$

we have $y \in f_{N}(x, t)$. Thus $f(x, t)$ is compact.

Assume that $f_{N}$ is not usc at $(x, t)$. There exists $\varepsilon>0$ such that for any $\delta>0$ there exist $y \in N, s \in \mathbb{R}$ such that

$$
d(x, y)<\delta,|t-s|<\delta, f_{N}(y, s) \nsubseteq B\left(f_{N}(x, t), \varepsilon\right) .
$$

For each positive integer $n$, there exist $x_{n} \in N, t_{n} \in \mathbb{R}$ such that

$$
d\left(x, x_{n}\right)<\frac{1}{n},|t-s|<\frac{1}{n}, f_{N}\left(x_{n}, t_{n}\right) \nsubseteq B\left(f_{N}(x, t), \varepsilon\right) .
$$

Let $y_{n} \in f_{N}\left(x_{n}, t_{n}\right)-B\left(f_{N}(x, t), \varepsilon\right)$. Since $N$ is compact, $\left(y_{n}\right)$ has a convergent subsequence. Let $y_{n} \rightarrow y \in N$. Since $x_{n} \rightarrow x, t_{n} \rightarrow t, y_{n} \rightarrow y$, and $\operatorname{conn}_{N}\left(t_{n}, x_{n}, y_{n}\right) \neq \emptyset$ for all $n$, by Proposition 2.12, we have $\operatorname{conn}_{N}(t, x, y) \neq \emptyset$. Thus $y \in f_{N}(x, t)$. This is a contradiction. Hence $f_{N}$ is usc. 
The verification of properties (1) to (3) of Definition 2.6 is straightforward.

Given a subset $N \subset X$, we introduce the following notation

$$
\begin{aligned}
i n v^{+} N & =\left\{x \in N \mid \operatorname{sol}_{N}\left(\mathbb{R}^{+}, x\right) \neq \emptyset\right\}, \\
i n v^{-} N & =\left\{x \in N \mid \operatorname{sol}_{N}\left(\mathbb{R}^{-}, x\right) \neq \emptyset\right\}, \\
i n v N & =\left\{x \in N \mid \operatorname{sol}_{N}(\mathbb{R}, x) \neq \emptyset\right\} .
\end{aligned}
$$

By Proposition 2.8, we have inv $N=i n v^{+} N \cap i n v^{-} N$.

Let $\operatorname{diam}_{N} f=\sup \{\operatorname{diam} f(x, t) \mid x \in N, 0 \leq t \leq 1\}$ and $\operatorname{dist}(A, B)=$ $\inf \{d(x, y) \mid x \in A, y \in B\}, A, B \subset X$. If $A \subset X$, we denote the boundary of $A$ by $\partial A$, its interior by int $A$, and let $B(A, \varepsilon)=\{x \in X \mid d(x, A)<\varepsilon\}$ for $\varepsilon>0$.

Definition 3.2. A compact set $N \subset X$ is called an isolating neighborhood for a dispersive dynamical system $f$ if

$$
B\left(\operatorname{inv} N, \operatorname{diam}_{N} f\right) \subset \operatorname{int} N,
$$

or equivalently

$$
\operatorname{dist}(\operatorname{inv} N, \partial N)>\operatorname{diam}_{N} f .
$$

Definition 3.3. Let $N$ be an isolating neighborhood for a dispersive dynamical system $f$. A pair $P=\left(P_{1}, P_{2}\right)$ of compact subsets $P_{2} \subset P_{1} \subset N$ is called an index pair if the following conditions are satisfied

(i) $f\left(P_{i}, t\right) \cap N \subset P_{i}, i=1,2,0 \leq t \leq 1$;

(ii) $f\left(P_{1}-P_{2}, t\right) \subset N, 0 \leq t \leq 1$;

(iii) $\operatorname{invN} \subset \operatorname{int}\left(P_{1}-P_{2}\right)$.

Our first aim is to prove the following result.

Theorem 3.4. Let $N$ be an isolating neighborhood for a dispersive dynamical system $f$ and $W$ a neighborhood of inv $N$. Then there exists an index pair $P=\left(P_{1}, P_{2}\right)$ for $N$ with $P_{1}-P_{2} \subset W$.

The proof is based on several lemmas. First, given $N \subset X, s \in N, \tau \in \mathbb{R}^{+}$, the following notation will be used

$$
\begin{aligned}
f_{N, \tau}(x) & =\left\{y \in N \mid \operatorname{conn}_{N}(\tau, x, y) \neq \emptyset\right\} \\
f_{N,-\tau}(x) & =\left\{y \in N \mid \operatorname{conn}_{N}(-\tau, x, y) \neq \emptyset\right\}, \\
f_{N}^{+}(x) & =\bigcup_{r \in \mathbb{R}^{+}} f_{N, \tau}(x), f_{N}^{-}(x)=\bigcup_{r \in \mathbb{R}^{+}} f_{N,-\tau}(x) .
\end{aligned}
$$

Lemma 3.5. If $N \subset X$ is compact, then the map $f_{N, \tau}: N \rightarrow 2^{N}$ is usc for any $\tau \in \mathbb{R}$.

Proof. It is enough to prove that the assertion for $\tau \in \mathbb{R}^{+}$since the case of a negative $\tau$ is analogous. Suppose that $f_{N, \tau}$ is not usc at $x \in N$. There exists $\varepsilon>0$ such that for each $\delta>0$ there exists $y \in N$ such that $d(x, y)<\delta$ and $f_{N, \tau}(y) \nsubseteq B\left(f_{N, \tau}(x), \varepsilon\right)$. For each positive integer $n$, there exists $x_{n} \in N$ 
such that $d\left(x, x_{n}\right)<\frac{1}{n}$ and $f_{N, \tau}\left(x_{n}\right) \nsubseteq B\left(f_{N, \tau}(x), \varepsilon\right)$. Let $y_{n} \in f_{N, \tau}\left(x_{n}\right)-$ $B\left(f_{N, \tau}(x), \varepsilon\right)$. There exists a solution $\sigma_{n}:[0, \tau] \rightarrow N$ such that $\sigma_{n}(0)=x_{n}$ and $\sigma_{n}(\tau)=y_{n}$. Since $\sigma_{n}(0)=x_{n} \rightarrow x$, by Barbashin's Theorem, there exist a subsequence $\left(\sigma_{n_{i}}\right)$ of $\left(\sigma_{n}\right)$ and a solution $\gamma:[0, \tau] \rightarrow X$ such that $\left(\sigma_{n_{i}}\right)$ converges uniformly to $\gamma$ on $[0, \tau]$. Clearly $\gamma:[0, \tau] \rightarrow N$. Since $N$ is compact, $\left(y_{n_{i}}\right)$ has a convergent subsequence. Let $y_{n_{i}} \rightarrow y \in N$. We have

$$
\begin{aligned}
& \gamma(0)=\lim _{i \rightarrow \infty} \sigma_{n_{i}}(0)=\lim _{i \rightarrow \infty} x_{n_{i}}=\lim _{n \rightarrow \infty} x_{n}=x, \\
& \gamma(\tau)=\lim _{i \rightarrow \infty} \sigma_{n_{i}}(\tau)=\lim _{i \rightarrow \infty} y_{n_{i}}=y .
\end{aligned}
$$

Thus $y \in f_{N, \tau}(x)$. Since $d\left(y_{n}, f_{N, \tau}(x)\right) \geq \varepsilon$ for all $i$, we have $d\left(y, f_{N, \tau}(x)\right) \geq \varepsilon$. This is a contradiction. Hence $f_{N, \tau}$ is usc.

Lemma 3.6. Suppose that $D\left(f_{N, \tau}\right) \equiv\left\{x \in N \mid f_{N, \tau}(x) \neq \emptyset\right\}$ are nonempty for all $\tau \in \mathbb{R}$. Then inv $N$ is nonempty. Moreover, inv ${ }^{+} N=\bigcap_{\tau \in \mathbb{R}^{+}} D\left(f_{N, \tau}\right)$ and $i n v^{-} N=\bigcap_{\tau \in \mathbb{R}^{-}} D\left(f_{N, \tau}\right)$.

Proof. Since $\left\{D\left(f_{N, \tau}\right) \mid \tau \in \mathbb{R}^{+}\right\}$is a family of nonempty closed sets with finite intersection property, $K=\cap_{\tau \in \mathbb{R}^{+}} D\left(f_{N, \tau}\right)$ is nonempty. We prove that $i n v^{+} N=K$. The proof for $i n v^{-} N$ is analogous and the conclusion for $i n v N$ follows from Proposition 2.8. The inclusion $i n v^{+} N \subset K$ is obvious. Let $x \in K$. For each positive integer $n$, there exists a solution $\sigma_{n}:[0, n] \rightarrow N$ such that $\sigma_{n}(0)=x$. By Proposition 2.13, there exists a solution $\sigma: \mathbb{R}^{+} \rightarrow N$ such that $\sigma(0)=x$. Thus $x \in i n v^{+} N$.

Lemma 3.7. If $N \subset X$ is compact, then

(a) the sets inv ${ }^{+} N$, inv ${ }^{-} N$ and inv $N$ are compact;

(b) if $A$ is compact with inv $v^{-} \subset A \subset N$, then $f_{N}^{+}(A)$ is compact.

Proof. (a) Obvious.

(b) It is sufficient to show that $f_{N}^{+}(A)$ is closed. Let $y \in \overline{f_{N}^{+}(A)}$. There exists a sequence $\left(y_{n}\right)$ in $f_{N}^{+}(A)$ such that $y_{n} \rightarrow y$. For each positive integer $n$, there exist $t_{n} \in \mathbb{R}^{+}$and a solution $\sigma_{n}:\left[0, t_{n}\right] \rightarrow N$ such that $\sigma_{n}\left(t_{n}\right)=y_{n}$ and $\sigma_{n}(0) \in A$.

Case $1:\left(t_{n}\right)$ is bounded. $\left(t_{n}\right)$ has a convergent subsequence. Let $t_{n} \rightarrow t \in$ $\mathbb{R}^{+}$. By Proposition 2.12, we have $y \in f_{N, t}(A) \subset f_{N}^{+}(A)$.

Case 2: $\left(t_{n}\right)$ is unbounded. We may assume that $t_{n} \rightarrow \infty$. For each positive integer $n$, define $\gamma_{n}:\left[-t_{n}, 0\right] \rightarrow N$ by $\gamma_{n}(t)=\sigma_{n}\left(t+t_{n}\right)$. Since $\gamma_{n}$ is a solution and $\gamma_{n}(0)=\sigma_{n}\left(t_{n}\right)=y_{n}$, we have $f_{N,-t_{n}}\left(y_{n}\right) \neq \emptyset$, that is $y_{n} \in D\left(f_{N,-t_{n}}\right)$. Thus we have

$$
y \in \bigcap_{n=1}^{\infty} D\left(f_{N,-t_{n}}\right)=i n v^{-} N \subset A \subset f_{N}^{+}(A) .
$$

Hence $f_{N}^{+}(A)$ is closed. 
Lemma 3.8. If $K \subset N$ is a compact subset of $X$ with $K \cap i n v^{+} N=\emptyset$ (respectively, $K \cap i n v^{-} N=\emptyset$ ), then

(a) there exists $T \in \mathbb{R}^{+}$(respectively, $\mathbb{R}^{-}$) such that $f_{N, \tau}(K)=\emptyset$ for all $\tau \geq T$ (respectively, $\tau \leq T$ )

(b) the map $f_{N}^{+}$(respectively $f_{N}^{-}$) is usc on $K$;

(c) $f_{N}^{+}(K) \cap i n v^{+} N=\emptyset$ (respectively, $\left.f_{N}^{-} \cap i n v^{-} N=\emptyset\right)$.

Proof. (a) Assume that $K \cap i n v^{+} N=\emptyset$. For each $x \in K$, since $x \notin i n v^{+} N$, by Lemma 3.6, there exists $t_{x} \in \mathbb{R}^{+}$such that $f_{N, t_{x}}(x)=\emptyset$. Since $f_{N, t_{x}}$ is usc, there exists a neighborhood $V_{x}$ of $x$ such that $f_{N, t_{x}}(y)=\emptyset$ for all $y \in V_{x}$. Then the family $\left\{V_{x} \mid x \in K\right\}$ is an open cover of $K$. Since $K$ is compact, there are finitely many $x_{1}, \ldots, x_{n} \in K$ such that $K \subset \cup_{i=1}^{n} V_{x_{i}}$. Let $T=\max t_{x_{i}}$. If $\tau \geq T$, then

$$
f_{N, \tau}(K) \subset f_{N, \tau}\left(\bigcup_{i=1}^{n} V_{x_{i}}\right)=\bigcup_{i=1}^{n} f_{N, \tau}\left(V_{x_{i}}\right) \subset \bigcup_{i=1}^{n} f_{N, t_{x_{i}}}\left(V_{x_{i}}\right)=\emptyset .
$$

Thus $f_{N, \tau}(K)=\emptyset$.

(b) For any open subset $U$ of $X,\left(f_{N}^{+}\right)^{-1}(U)=\cup_{\tau \in \mathbb{R}^{+}}\left(f_{N, \tau}\right)^{-1}(U)$ is open. Thus $f_{N}^{+}$is usc.

(c) Let $x \in f_{N}^{+}(K) \cap i n v^{+} N$. Since $x \in f_{N}^{+}(K)$, there exist $\tau \in \mathbb{R}^{+}$and a solution $\sigma_{1}:[0, \tau] \rightarrow N$ such that $\sigma_{1}(\tau)=x$ and $\sigma_{1}(0) \in K$. Since $x \in i n v^{+} N$, there exists a solution $\sigma_{2}: \mathbb{R}^{+} \rightarrow N$ such that $\sigma_{2}(0)=x$. Define $\sigma: \mathbb{R}^{+} \rightarrow N$ by

$$
\sigma(t)=\left\{\begin{array}{lc}
\sigma_{1}(t), & 0 \leq t \leq \tau \\
\sigma_{2}(t-\tau), & t \geq \tau
\end{array}\right.
$$

Since $\sigma$ is a solution and $\sigma(0)=\sigma_{1}(0) \in K$, we have $\sigma(T) \in f_{N, T}(K) \cap i n v^{+} N$. This is a contradiction. Thus $f_{N}^{+}(K) \cap i n v^{+} N=\emptyset$.

Lemma 3.9. If $N$ is a compact subset of $X$, then for any neighborhood $V$ of $i n v^{-} N$ there exists a compact neighborhood $A$ of inv ${ }^{-} N$ such that $f_{N}^{+}(A) \subset V$.

Proof. Since $N-V$ is compact and $(N-V) \cap i n v^{-} N=\emptyset$, by Lemma 3.8, there exists $T \in \mathbb{R}^{+}$such that $f_{N,-T}(N-V)=\emptyset$. For each $x \in i n v^{-} N$, since $f_{N, T}$ is usc, there exists a compact neighborhood $V_{x}$ of $x$ such that $f_{N, T}\left(V_{x}\right) \subset V$. Since $i n v^{-} N$ is compact, there are finitely many points $x_{1}, \ldots, x_{n} \in i n v^{-} N$ such that $K \subset \cup_{i=1}^{n} V_{x_{i}}$. Let $A=\cup_{i=1}^{n} V_{x_{i}}$. Then $A$ is a compact neighborhood of $i n v^{-} N$ and

$$
f_{N, T}(A) \subset f_{N, T}\left(\bigcup_{i=1}^{n} V_{x_{i}}\right)=\bigcup_{i=1}^{n} f_{N, T}\left(V_{x_{i}}\right) \subset V .
$$

Let $y \in f_{N}^{+}(A)$. Then there exist $\tau \in \mathbb{R}^{+}$and $x \in A$ such that $y \in f_{N, \tau}(x)$. If $\tau \leq T$, then

$$
y \in f_{N, \tau}(x) \subset f_{N, \tau}(A) \subset f_{N, T}(A) \subset V .
$$


If $\tau>T$, then we have $x \in f_{N,-\tau}(y) \subset f_{N,-T}(y)$. Since $f_{N,-T}(N-V)=\emptyset$, we have $y \in V$. Thus $f_{N}^{+}(A) \subset V$.

Proof of Theorem 3.4. Since $N$ is an isolating neighborhood, we have

$$
\operatorname{inv} N \subset B\left(i n v N, \operatorname{diam}_{N} f\right) \subset i n v N .
$$

Thus we may assume that $W \subset \operatorname{int} N$. Choose $0<\varepsilon<\operatorname{dist}(\operatorname{inv} N, \partial N)-$ $\operatorname{diam}_{n} f$ and let $\gamma=\varepsilon+\operatorname{diam}_{N} f$. Since $\gamma<\operatorname{dist}(\operatorname{inv} N, \partial N)$, we have $B(i n v N, \gamma)$ $\subset \operatorname{int} N$. Since the set $\{x \in X \mid f(x,[0,1]) \subset B(\operatorname{inv} N, \gamma)\}$ is an open neighborhood of $i n v N$, we may assume that $f(W, t) \subset \operatorname{int} N$ for all $0 \leq t \leq 1$. Let $U$ and $V$ be open neighborhoods of $i n v^{+} N$ and $i n v^{-} N$, respectively, such that $U \cap V \subset W$. By Lemma 3.9, there exists a compact neighborhood $A$ of $i n v^{-} N$ such that $f_{N}^{+}(A) \subset V$. We define

$$
P_{1}=f_{N}^{+}(A), P_{2}=f_{N}^{+}\left(P_{1}-U\right) .
$$

Then $P_{1} \subset V$ and $P_{1}-U \subset P_{2}$ which implies that $P_{1}-P_{2} \subset U$. Thus $P_{1}-P_{2} \subset$ $U \cap V \subset W$. We verify that $\left(P_{1}, P_{2}\right)$ is an index pair. By Lemma 3.7, $P_{1}$ is compact. Since $P_{1}-U$ is compact, by Lemma 3.7, $P_{2}$ is compact. We have

$$
P_{2}=f_{N}^{+}\left(P_{1}-U\right) \subset f_{N}^{+}\left(P_{1}\right)=f_{N}^{+}\left(f_{N}^{+}(A)\right)=f_{N}^{+}(A)=P_{1} .
$$

To verify (i), let $x \in P_{i}$ and $y \in f(x, t) \cap N$. Since $x \in P_{i}$, there exist $\tau \in \mathbb{R}^{+}$ and a solution $\sigma_{1}:[0, \tau] \rightarrow N$ such that $\sigma_{1}(\tau)=x$ and $\sigma_{1}(0) \in A$ in the case of $i=1, \sigma_{1}(0) \in P_{1}-U$ in the case $i=2$. There exists a solution $\sigma_{2}:[0, t] \rightarrow N$ such that $\sigma_{2}(0)=x$ and $\sigma_{2}(t)=y$. Define $\gamma:[0, \tau+t] \rightarrow N$ by

$$
\gamma(s)= \begin{cases}\sigma_{1}(s), & 0 \leq s \leq \tau \\ \sigma_{2}(s-\tau), & \tau \leq s \leq \tau+t\end{cases}
$$

Then $\gamma$ is a solution and $\gamma(0)=\sigma_{1}(0), \gamma(\tau+t)=\sigma_{2}(t)=y$. Thus $y \in P_{i}$. Since $P_{1}-P_{2} \subset W$, we have $f\left(P_{1}-P_{2}, t\right) \subset f(W, t) \subset N$ for all $0 \leq t \leq 1$. Thus (ii) is verified. In order to verify (iii), observe that $P_{1}$ is a neighborhood of $i n v^{-} N$. Since $P_{1}-U$ is compact and $\left(P_{1}-U\right) \cap i n v^{+} N=\emptyset$, by Lemma 3.8,

$$
P_{2} \cap i n v^{+} N=f_{N}^{+}\left(P_{1}-U\right) \cap i n v^{+} N=\emptyset .
$$

Thus $N-P_{2}$ is a neighborhood of $i n v^{+} N$. Hence $P_{1}-P_{2}=P_{1} \cap\left(N-P_{2}\right)$ is a neighborhood of $i n v^{-} N \cap i n v^{+} N=i n v N$.

We shall now discuss several properties of index pairs which will be used in next section. In the remainder in this section, we denote $f(x, 1)$ by $F(x)$.

Proposition 3.10. (a) If $P$ is an index pair for $N$, then $\left(P_{1} \cap F\left(P_{2}\right)\right)-\left(P_{2} \cap\right.$ $\left.F\left(P_{2}\right)\right)=P_{1}-P_{2}$.

(b) If $P$ and $Q$ are index pairs for $N$, then so is $P \cap Q$.

(c) If $P \subset Q$ are index pairs for $N$, then so are $\left(P_{1}, P_{1} \cap Q_{2}\right)$ and $\left(P_{1} \cup\right.$ $\left.Q_{2}, Q_{2}\right)$ 
Proof. (a) We have $\left(P_{1} \cup F\left(P_{2}\right)\right)-\left(P_{2} \cup F\left(P_{2}\right)\right)=P_{1}-\left(P_{2} \cup F\left(P_{2}\right)\right) \subset P_{1}-P_{2}$. Let $x \in P_{1}-P_{2}$. If $x \in F\left(P_{2}\right)$, then we have $x \in F\left(P_{2}\right) \cap N \subset P_{2}$. This is a contradiction. Thus $x \in\left(P_{1} \cup F\left(P_{2}\right)\right)-\left(P_{2} \cup F\left(P_{2}\right)\right)$.

(b) Verification of (i) and (ii) is obvious. For (iii), let us note that

$$
\begin{aligned}
\operatorname{int}\left(P_{1}-P_{2}\right) \cap \operatorname{int}\left(Q_{1}-Q_{2}\right) & \subset \operatorname{int}\left(P_{1} \cap Q_{1}-\left(P_{2} \cap Q_{2}\right)\right) \\
& \subset \operatorname{int}\left(P_{1} \cap Q_{1}-\left(P_{2} \cup Q_{2}\right)\right) .
\end{aligned}
$$

(c) Obvious.

Proposition 3.11. Let $P \subset Q$ be index pairs for $N$ such that $P_{1}=Q_{1}$ or $P_{2}=Q_{2}$. Define a pair of sets $G(P, Q)$ by

$$
G_{i}(P, Q)=P_{i} \cup\left(F\left(Q_{i}\right) \cap N\right), i=1,2 .
$$

Then (a) if $P_{i}=Q_{i}$, then $G_{i}(P, Q)=P_{i}=Q_{i}, i=1,2$;

(b) $P \subset G(P, Q) \subset Q$;

(c) $G(P, Q)$ is an index pair;

(d) $F\left(Q_{i}\right) \cap N \subset G_{i}(P, Q), i=1,2$.

Proof. (a), (b) and (d) are clear. It remains to prove that (c). For (i), let $x \in G_{i}(P, Q), y \in F(x) \cap N$. If $x \in P_{i}$, then $y \in F\left(P_{i}\right) \cap N \subset P_{i} \subset G_{i}(P, Q)$. If $x \in F\left(Q_{i}\right) \cap N \subset Q_{i}$, then $y \in F\left(Q_{i}\right) \cap N \subset P_{i} \subset G_{i}(P, Q)$. For (ii), since $G_{1}(P, Q)=P_{1} \cup\left(F\left(Q_{1}\right) \cap N\right) \subset P_{1} \cup Q_{1}=Q_{1}, P_{2} \subset G_{2}(P, Q)$, we have

$$
G_{1}(P, Q)-G_{2}(P, Q) \subset Q_{1}-P_{2} .
$$

If $P_{1}=Q_{1}$, we have $F\left(G_{1}(P, Q)-G_{2}(P, Q)\right) \subset F\left(Q_{1}-P_{2}\right)=F\left(P_{1}-P_{2}\right) \subset N$. If $P_{2}=Q_{2}$, we have $F\left(G_{1}(P, Q)-G_{2}(P, Q)\right) \subset F\left(Q_{1}-P_{2}\right)=F\left(Q_{1}-Q_{2}\right) \subset N$. For (iii), we show that $\left(P_{1}-P_{2}\right) \cap\left(Q_{1}-Q_{2}\right) \subset G_{1}(P, Q)-G_{2}(P, Q)$. Let $y \in\left(P_{1}-P_{2}\right) \cap\left(Q_{1}-Q_{2}\right)$. Since $P_{1} \subset G_{1}(P, Q)$ and $G_{2}(P, Q)=P_{2} \cup\left(F\left(Q_{2}\right) \cap\right.$ $N) \subset P_{2} \cup Q_{2}=Q_{2}$, we have $y \in G_{1}(P, Q)$ and

$$
\begin{aligned}
\operatorname{invN} & \subset \operatorname{int}\left(P_{1}-P_{2}\right) \cap i n t\left(Q_{1}-Q_{2}\right) \\
& \subset \operatorname{int}\left(\left(P_{1}-P_{2}\right) \cap\left(Q_{1}-Q_{2}\right)\right) \\
& \subset \operatorname{int}\left(G_{1}(P, Q)-G_{2}(P, Q)\right) .
\end{aligned}
$$

Proposition 3.12. Let $P \subset Q$ be index pairs such that $P_{1}=Q_{1}$ or $P_{2}=Q_{2}$. Then there exists a sequence of pairs

$$
P=Q^{n} \subset Q^{n-1} \subset \cdots \subset Q^{1} \subset Q^{0}=Q
$$

with the following properties:

(a) if $P_{i}=Q_{i}$, then $Q_{i}^{k}=P_{i}=Q_{i}$ for all $k=1,2, \ldots, n-1, i=1,2$;

(b) $Q^{k}$ is an index pair for all $k=1,2, \ldots, n-1$;

(c) $F\left(Q_{i}^{k}\right) \cap N \subset Q_{i}^{k+1}, i=1,2, k=0,1, \ldots, n-1$.

Proof. We denote $Q^{0}=Q, Q^{k+1}=G\left(P, Q^{k}\right)$. By Proposition 3.11, $\left(Q^{k}\right)$ is a decreasing sequence of index pairs containing $P$ and satisfying (a), (b) and (c). It remains to show that $Q^{n}=P$ for some $n$. Suppose that the inclusion $P \subset Q^{k}$ is strict for all $k$. We may assume that $Q_{2}^{k} \neq P_{2}$ for all $k$. For any 
positive integer $k$, choose $\sigma(k) \in Q_{2}^{k}-P_{2}$. Since $\sigma(k) \in F\left(Q_{2}^{k-1}\right) \cap N$, there exists $\sigma(k-1) \in Q_{2}^{k-1}$ such that $\sigma(k) \in F(\sigma(k-1))$. If $\sigma(k-1) \in P_{2}$, then $\sigma(k) \in F\left(P_{2}\right) \cap N \subset P_{2}$. This is a contradiction. Thus $\sigma(k-1) \in Q_{2}^{k-1}-P_{2}$. Repeating this process, we have solution $\sigma_{k}:[0, k] \rightarrow Q_{2}-i n t P_{2}$ such that $\sigma_{k}(i)=\sigma(i), 0 \leq i \leq k$. Since $D\left(f_{Q_{2}-i n t P_{2}, k}\right) \neq \emptyset$ for all $k$, by Lemma 3.6, we have $\emptyset \neq i n v\left(Q_{2}-i n t P_{2}\right) \subset i n v Q_{2}$. On the other hand inv $Q_{2} \subset Q_{2}$ and inv $Q_{2} \subset \operatorname{int}\left(Q_{1}-Q_{2}\right) \subset Q_{1}-Q_{2}$ implies that inv $Q_{2}=\emptyset$, a contradiction.

\section{Existence of stable index pairs}

Let $(X, d)$ be a locally compact metric space and $\Omega(X)$ the set of all continuous dispersive dynamical systems on $X$. Define a metric $\rho: \Omega(X) \times \Omega(X) \rightarrow \mathbb{R}$ by

$$
\rho(f, g)=\sup \left\{\max \left\{\frac{1}{|t|} D(f(x, t), g(x, t)) \mid x \in X\right\} \mid t \neq 0\right\}
$$

for all $f, g \in \Omega(X)$, where $D$ is the Hausdorff metric induced by $d$. Let $\Lambda \subset \mathbb{R}$ be a compact interval.

Definition 4.1. A map $\phi: \Lambda \rightarrow \Omega(X)$ will be called a parametrized family of dispersive dynamical systems if the map $(\lambda, x, t) \in \Lambda \times X \times \mathbb{R} \mapsto \phi(\lambda)(x, t) \in 2^{X}$ is usc.

Define a map $\psi: \Lambda \times X \times \mathbb{R} \rightarrow 2^{\Lambda \times X}$ by $\psi(\lambda, x, t)=\{\lambda\} \times \phi(\lambda)(x, t)$. It is easy to show that $\psi$ is a dispersive dynamical system on $\Lambda \times X$.

Given a compact set $N \subset X$ and $\lambda \in \Lambda$, the sets $i n v^{( \pm)} N$ with respect to $\phi(\lambda)$ are denoted by $i n v^{( \pm)}(N, \lambda)$.

We need the following propositions to prove the existence of an stable index pair.

Proposition 4.2. Let $N \subset X$ be compact. Then the mappings

$$
\begin{aligned}
& \lambda \in \Lambda \quad \mapsto \quad i n v^{+}(N, \lambda) \in 2^{N}, \\
& \lambda \in \Lambda \quad \mapsto \quad i n v^{-}(N, \lambda) \in 2^{N}, \\
& \lambda \in \Lambda \quad \mapsto \quad \operatorname{inv}(N, \lambda) \in 2^{N}
\end{aligned}
$$

are usc.

Proof. We prove the assertion for the first map only, since the other two proofs are by extending the same argument to negative numbers. Assume that the map

$$
\lambda \in \Lambda \mapsto i n v^{+}(N, \lambda) \in 2^{N}
$$

is not usc at $\lambda_{0} \in \Lambda$. Then there exist a neighborhood $U$ of $i n v^{+}\left(N, \lambda_{0}\right)$ and a sequence $\left(\lambda_{n}\right)$ in $\Lambda$ such that $\lambda_{n} \rightarrow \lambda_{0}$ and $i n v^{+}\left(N, \lambda_{n}\right) \nsubseteq$ for all $n$. Choose $x_{n} \in i n v^{+}\left(N, \lambda_{n}\right)-U$ and

$$
\sigma_{n} \in \operatorname{sol}_{N}\left(\mathbb{R}^{+}, x_{n}, \lambda_{n}\right) \subset \operatorname{sol}_{\Lambda \times N}\left(\mathbb{R}^{+},\left(\lambda_{n}, x_{n}\right)\right) .
$$


Since $N$ is compact, $\left(x_{n}\right)$ has a convergent subsequence. Let $x_{n} \rightarrow x \in N-$ $U$. By Proposition 2.12, there exists $\sigma \in \operatorname{sol}_{\Lambda \times N}\left(\mathbb{R}^{+},\left(\lambda_{0}, x\right)\right)$. Since $\sigma(0)=$ $\left(\lambda_{0}, x\right) \in\left\{\lambda_{0}\right\} \times N$, we have $\sigma \in \operatorname{sol}_{N}\left(\mathbb{R}^{+}, x, \lambda_{0}\right)$. Thus $x \in \operatorname{inv}^{+}\left(N, \lambda_{0}\right) \subset U$, which contradicts $x \notin U$.

Proposition 4.3. Let $\lambda_{0} \in \Lambda$ and let $N$ be an isolating neighborhood for $\phi\left(\lambda_{0}\right)$. Then $N$ is an isolating neighborhood for $\phi(\lambda)$ for all $\lambda$ sufficiently close to $\lambda_{0}$.

Proof. Since $\operatorname{dist}\left(\operatorname{inv}\left(N, \lambda_{0}\right), \partial N\right)>\operatorname{diam}_{N} \phi\left(\lambda_{0}\right)$, choose $\varepsilon$ such that

$$
0<3 \varepsilon<\operatorname{dist}\left(\operatorname{inv}\left(N, \lambda_{0}\right), \partial N\right)-\operatorname{diam}_{N} \phi\left(\lambda_{0}\right) .
$$

Then $B\left(\operatorname{inv}\left(N, \lambda_{0}\right), \operatorname{diam}_{N} \phi\left(\lambda_{0}\right)+3 \varepsilon\right) \subset \operatorname{int} N$. Since the map

$$
(\lambda, x, t) \in \Lambda \times X \times \mathbb{R} \mapsto \phi(\lambda)(x, t) \in 2^{X}
$$

is usc and $N$ is compact, $\phi(\lambda)(x, t) \subset B\left(\phi\left(\lambda_{0}\right)(x, t), \varepsilon\right)$ for all $\lambda$ close to $\lambda_{0}$, all $x \in N$ and all $0 \leq t \leq 1$. By compactness of $N$, $\operatorname{diam}_{N} \phi(\lambda)<\operatorname{diam}_{N} \phi\left(\lambda_{0}\right)+2 \varepsilon$ for all $\lambda$ close to $\lambda_{0}$. By Proposition 4.2, $\operatorname{inv}(N, \lambda) \subset B\left(\operatorname{inv}\left(N, \lambda_{0}\right), \varepsilon\right)$ for all $\lambda$ close to $\lambda_{0}$ and we get

$$
\begin{aligned}
B\left(\operatorname{inv}(N, \lambda), \operatorname{diam}_{N} \phi(\lambda)\right) & \subset B\left(B\left(i n v\left(N, \lambda_{0}\right), \varepsilon\right), \operatorname{diam}_{N} \phi\left(\lambda_{0}\right)+2 \varepsilon\right) \\
& =B\left(i n v\left(N, \lambda_{0}\right), \operatorname{diam}_{N} \phi\left(\lambda_{0}\right)+3 \varepsilon\right) \\
& \subset \operatorname{int} N .
\end{aligned}
$$

Proposition 4.4. Let $f: X \times \mathbb{R} \rightarrow 2^{X}$ be a dispersive dynamical system, $N$ an isolating neighborhood for $f$ and $P$ an index pair for $N$ and $f$. If $g: X \times \mathbb{R} \rightarrow 2^{X}$ is a dispersive dynamical system such that $f(x, t) \subset f(x, t)$ for all $(x, t) \in X \times \mathbb{R}$, then $N$ is an isolating neighborhood for $g$, inv ${ }^{( \pm)}(N, g) \subset i n v^{( \pm)}(N, f)$ and $P$ is also an index pair for $N$ and $g$.

Proof. It is clear.

The main result of this section is the following.

Theorem 4.5. Let $f: X \times \mathbb{R} \rightarrow 2^{X}$ be a continuous dispersive dynamical system, $N$ an isolating neighborhood for $f$ and $W$ an open neighborhood of inv $N$. Then there exists an index pair $P=\left(P_{1}, P_{2}\right)$ for $N$ with $P_{1}-P_{2} \subset W$ which is stable under small continuous perturbations of $f$, i.e., there exists $\varepsilon>0$ such that if $g \in B_{\rho}(f, \varepsilon)$, then $P$ also is an index pair for $g$.

Proof. Define a family of dispersive dynamical systems $\phi:[0,1] \rightarrow \Omega(X)$ by

$$
\begin{aligned}
& \phi_{\lambda}(x, t)=\bar{B}(f(x, t), \lambda t) \text { for } x \in X, t \in \mathbb{R}^{+}, \\
& \phi_{\lambda}(x, t)=\left\{y \in X \mid x \in \phi_{\lambda}(y,-t)\right\} \text { for } x \in X, t \in \mathbb{R}^{-} .
\end{aligned}
$$

By Propositions 4.2 and 4.3, there exists $\tau>0$ such that $N$ is an isolating neighborhood for $\phi_{\lambda}$ and $\operatorname{inv}(N, \lambda) \subset W$ provided $0 \leq \lambda \leq \tau$. Define

$$
P_{1}=i n v^{-}(N, \tau), P_{2}=P_{1}-i n t\left(i n v^{+}(N, \tau)\right) .
$$


Note that $\overline{P_{1}-P_{2}}=\operatorname{inv}(N, \tau) \subset W$. We shall verify below that $P=\left(P_{1}, P_{2}\right)$ is an index pair for all $\phi_{\lambda}$ with $0 \leq \lambda<\tau$. In particular, it is an index pair for $\phi(0)=f$. Moreover, if $g \in B_{\rho}(f, \varepsilon)$ for $\varepsilon<\tau$, then $g(x, t) \subset \phi_{\tau}(x, t)$ for all $(x, t) \in X \times \mathbb{R}$. Thus the conclusion follows from Proposition 4.4. We will show that $P$ is an index pair for $\phi_{\lambda}$ provided $0 \leq \lambda<\tau$.

(i) $\phi_{\lambda}\left(P_{i}, t\right) \cap N \subset P_{i}, i=1,2,0 \leq t \leq 1$.

Let $y \in \phi_{\lambda}\left(P_{1}, t\right) \cap N$. Then there exists $x \in P_{1}$ such that $y \in \phi_{\lambda}(x, t)$. Since $P_{1}=i n v^{-}(N, \tau)$, there exists a solution $\sigma_{1}: \mathbb{R}^{-} \rightarrow N$ for $\phi_{\tau}$ such that $\sigma_{1}(0)=x$. Also there exists a solution $\sigma_{2}:[0, t] \rightarrow N$ for $\phi_{\lambda}$ such that $\sigma_{2}(0)=x, \sigma_{2}(t)=y$. Since

$$
\sigma_{2}(s) \in \phi_{\lambda}(x, s) \subset \phi_{\tau}(x, s)
$$

for all $0 \leq s \leq t, \sigma_{2}$ is a solution for $\phi_{\tau}$. Define $\sigma: \mathbb{R}^{-} \rightarrow N$ by

$$
\sigma(s)= \begin{cases}\sigma_{2}(s+t), & -t \leq s \leq 0 \\ \sigma_{1}(s+t), & s \leq t\end{cases}
$$

Then $\sigma$ is a solution for $\phi_{\tau}$ and since $\sigma(0)=y$, we have $y \in i n v^{-}(N, \tau)=$ $P_{1}$. Let $y \in \phi_{\lambda}\left(P_{2}, t\right) \cap N$. Then there exists $x \in P_{2}$ such that $y \in \phi_{\lambda}(s, t)$. Since $x \in P_{1}$, we have $y \in P_{1}$. In order to show that $y \notin \operatorname{int}\left(\operatorname{inv}^{+}(N, \tau)\right)$, suppose that $y \in \operatorname{int}\left(i n v^{+}(N, \tau)\right)$. Then there exists $\varepsilon>0$ such that $B(y, \varepsilon) \subset$ $i n v^{+}(N, \tau)$. Since $\phi_{\tau} \in \Omega(X)$, if $d(x, z)<\varepsilon$, then there exists $\delta>0$ such that $\phi_{\tau}(x, t) \subset B\left(\phi_{\tau}(z, t), \varepsilon\right)$. Since $y \in \phi_{\lambda}(x, t) \subset \phi_{\tau}(x, t)$ for $z \in B(x, \delta)$, there exists $p \in \phi_{\tau}(z, t)$ such that $d(y, p)<\varepsilon$. Since $p \in B(y, \varepsilon) \subset i n v^{+}(N, \tau)$, there exists a solution $\sigma_{1}: \mathbb{R}^{+} \rightarrow N$ for $\phi_{\tau}$ such that $\phi_{1}(0)=p$. Since $p \in \phi_{\tau}(z, t)$, there exists a solution $\sigma_{2}:[0, t] \rightarrow N$ for $\phi_{\tau}$ such that $\sigma_{2}(0)=z, \sigma_{2}(t)=p$. Define $\sigma: \mathbb{R}^{+} \rightarrow N$ by

$$
\sigma(s)= \begin{cases}\sigma_{2}(s), & 0 \leq s \leq t \\ \sigma_{1}(s-t), & s \geq t .\end{cases}
$$

Then $\sigma$ is a solution for $\phi_{\tau}$. Since $\sigma(0)=z$, we have $z \in i n v^{+}(N, \tau)$, that is, $B(x, \delta) \subset i n v^{+}(N, \tau)$. Therefore we have $x \in \operatorname{int}\left(i n v^{+}(N, \tau)\right)$. This contradicts $x \in P_{2}$.

(ii) $\phi_{\lambda}\left(P_{1}-P_{2}, t\right) \subset N$.

Since $\phi_{\lambda}\left(P_{1}-P_{2}, t\right) \subset \phi_{\tau}\left(P_{1}-P_{2}, t\right) \subset N$, it holds.

(iii) $\operatorname{inv}(N, \lambda) \subset \operatorname{int}\left(P_{1}-P_{2}\right)$.

Since $\operatorname{int}\left(P_{1}-P_{2}\right)=\operatorname{int}(\operatorname{inv}(N, \tau))$, we show only that the following holds;

$$
0 \leq \lambda<\tau \text { implies } i n v^{ \pm}(N, \lambda) \subset \operatorname{int}\left(i n v^{ \pm}(N, \tau)\right) .
$$

Since $\tau-\lambda>0$, if $d(x, z)<\delta$, then there exists $\delta>0$ such that $f(x, 1) \subset$ $B(f(z, 1), \tau-\lambda)$. We have

$$
\phi_{\lambda}(x, 1)=\bar{B}(f(x, 1), \lambda) \subset \bar{B}(B(f(z, 1), \tau-\lambda), \lambda)=\bar{B}(f(z, 1), \tau)=\phi_{\tau}(z, 1) .
$$

Let $x \in i n v^{+}(N, \lambda)$. Then there exists a solution $\sigma_{1}: \mathbb{R}^{+} \rightarrow N$ for $\phi_{\lambda}$ such that $\sigma_{1}(0)=x$. For any $z \in B(x, \delta)$, let $y \in \phi_{\lambda}(x, 1) \subset \phi_{\tau}(z, 1)$. Then there exist a 
solution $\sigma_{2}:[0,1] \rightarrow N$ for $\phi_{\lambda}$ and a solution $\sigma_{3}:[0,1] \rightarrow N$ for $\phi_{\tau}$ such that $\sigma_{2}(0)=x, \sigma_{2}(1)=y$ and $\sigma_{3}(0)=z, \sigma_{3}(1)=y$. Define $\sigma: \mathbb{R}^{+} \rightarrow N$ by

$$
\sigma(s)= \begin{cases}\sigma_{3}(s), & 0 \leq s \leq 1 \\ \sigma_{2}(2-s), & 1 \leq s \leq 2 \\ \sigma_{1}(s-2), & s \geq 2\end{cases}
$$

Then $\sigma$ is a solution for $\phi_{\tau}$. Since $\sigma(0)=z$, we have $z \in i n v^{+}(N, \tau)$. Thus we have

$$
B\left(i n v^{+}(N, \lambda), \delta\right) \subset i n v^{+}(N, \tau)
$$

and so it follows that $i n v^{+}(N, \lambda) \subset \operatorname{int}\left(i n v^{+}(N, \tau)\right)$.

Let $x \in i n v^{-}(N, \lambda)$. Then there exists a solution $\sigma_{1}: \mathbb{R}^{-} \rightarrow N$ for $\phi_{\lambda}$ such that $\sigma_{1}(0)=x$. Let $\sigma(-1)=y$. Then we have $x=\sigma(0) \in \phi_{\lambda}(\sigma(-1), 1)=$ $\phi_{\lambda}(y, 1)$. For any $z \in B(x, \tau-\lambda)$, we have

$$
z \in \bar{B}\left(\phi_{\lambda}(y, 1), \tau-\lambda\right)=\bar{B}(\bar{B}(f(y, 1), \lambda), \tau-\lambda)=\bar{B}(f(y, 1), \tau)=\phi_{\tau}(y, 1) .
$$

Therefore there exists a solution $\sigma_{2}:[0,1] \rightarrow N$ for $\phi_{\tau}$ such that $\sigma_{2}(0)=$ $y, \sigma_{2}(1)=z$. Define $\sigma: \mathbb{R}^{-} \rightarrow N$ by

$$
\sigma(s)= \begin{cases}\sigma_{2}(s+1), & -1 \leq s \leq 0 \\ \sigma_{1}(s+1), & s \leq-1\end{cases}
$$

Then $\sigma$ is a solution for $\phi_{\tau}$. Since $\sigma(0)=z$, we have $z \in i n v^{-}(N, \tau)$. Hence we have

$$
B\left(i n v^{-}(N, \lambda), \tau-\lambda\right) \subset i n v^{-}(N, \tau) .
$$

It follows that $i n v^{-}(N, \lambda) \subset \operatorname{int}\left(i n v^{-}(N, \tau)\right)$.

\section{References}

[1] R. Churchill, Isolated invariant sets in compact metric spaces, J. Differential Equations 12 (1972), 330-352.

[2] C. C. Conley, Isolated Invariant Sets and the Morse Index, CBMS Regional Conf. Ser. Math. No. 38, Amer. Math. Soc., Providence RI, 1978.

[3] E. N. Dancer, Perturbation of zeros in the presence of symmetries, J. Austral. Math. Soc. Ser. A 36 (1984), no. 1, 106-125.

[4] _ Degenerate critical points, homotopy indices and Morse inequalities, J. Reine Angew. Math. 350 (1984), 1-22.

[5] A. Dold, Fixed point index and fixed point theorem for Euclidean neighborhood retracts, Topology 4 (1965), 1-8.

[6] T. Kaczynski and M. Morzec, Conley index for discrete multi-valued dynamical systems, Topology Appl. 65 (1995), no. 1, 83-96.

[7] _ Stable index pairs for discrete dynamical systems, Canad. Math. Bull. 40 (1997), no. 4, 448-455.

[8] P. E. Kloeden, Asymptotic invariance and limit sets of general control systems, J. Differential Equations 19 (1975), no. 1, 91-105.

[9] H. L. Kurland, The Morse index of an isolated invariant set is a connected simple system, J. Differential Equations 42 (1981), no. 2, 234-259.

[10] D. Li, On dynamical stability in general dynamical systems, J. Math. Anal. Appl. 263 (2001), no. 2, 455-478. 
[11] J. T. Montgomery, Cohomology of isolated invariant sets under perturbation, J. Differential Equations 13 (1973), 257-299.

[12] E. Roxin, Stability in general control systems, J. Differential Equations 1 (1965), 115150.

[13] K. Rybakowski, On the homotopy index for infinite dimensional semiflows, Trans. Amer. Math. Soc. 269 (1982), no. 2, 351-382.

[14] K. S. Sibirsky, Introduction to Topological Dynamics, Noordhoff International Publishing, Leiden, 1975.

[15] H. Siegberg and G. Skordev, Fixed point index and chain approximations, Pacific J. Math. 102 (1982), no. 2, 455-486.

Yoon Hoe Goo

Department of Mathematics

Hanseo University

Chungnam 356-706, Korea

E-mail address: yhgoo@hanseo.ac.kr

JONG-SUH PARK

Department of Mathematics

Chungnam National University

DAEJEON 305-764, KoreA

E-mail address: jspark@math.cnu.ac.kr 